\title{
Robust Filtering for Stochastic Genetic Regulatory Networks with Time-Varying Delay
}

\author{
Guoliang Wei ${ }^{a}$, Zidong Wang ${ }^{a, b, *}$, James Lam $^{c}$, Karl Fraser $^{b}$, Ganti Prasada Rao $^{d}$ \\ and Xiaohui $\mathrm{Liu}^{b}$
}

\begin{abstract}
This paper addresses the robust filtering problem for a class of linear genetic regulatory networks (GRNs) with stochastic disturbances, parameter uncertainties and time delays. The parameter uncertainties are assumed to reside in a polytopic region, the stochastic disturbance is state-dependent described by a scalar Brownian motion, and the time-varying delays enter into both the translation process and the feedback regulation process. We aim to estimate the true concentrations of mRNA and protein by designing a linear filter such that, for all admissible time delays, stochastic disturbances as well as polytopic uncertainties, the augmented state estimation dynamics is exponentially mean square stable with an expected decay rate. A delay-dependent linear matrix inequality (LMI) approach is first developed to derive sufficient conditions that guarantee the exponential stability of the augmented dynamics, and then the filter gains are parameterized in terms of the solution to a set of LMIs. Note that LMIs can be easily solved by using standard software packages. A simulation example is exploited in order to illustrate the effectiveness of the proposed design procedures.
\end{abstract}

\section{Keywords}

Genetic regulatory network; polytopic-type uncertainty; decay rate; time-varying delay; stochastic disturbance.

\section{INTRODUCTION}

In a living cell, the mechanisms that genes encode proteins and some of which in turn regulate gene expression construct a complex network, which is called Genetic Regulatory Networks (GRNs). With the appearance and development of DNA microarray technology [27], it has become possible to measure gene expression levels on a genomic scale and furthermore analyze the gene regulatory network. Considerable attention has been contributed to theoretical analysis and experimental investigation on GRNs and a large amount of results have been reported on dynamical behaviors of GRNs, see e.g. [1,7,13,19, 20,28].

During the past few years, modeling of genetic regulatory network has become an attractive area and a variety of models have been proposed, for example, Boolean network model $[15,22,23,32]$, linear differential equation model $[6,9,16]$ and a single negative feedback loop network [1]. It has been shown in [25], by mathematically modelling recent data, that the observed oscillatory expression and activity of three proteins is most likely to be driven by transcriptional delays, and time delay is often inevitable when analyzing the dynamical behaviors of GRNs [7,30,31]. On the other hand, the stochastic fluctuations in real-world gene expression data stem from either the probabilistic chemical reactions or random variation of one or more of

This work was supported in part by the Biotechnology and Biological Sciences Research Council (BBSRC) of the U.K. under Grants BB/C506264/1 and 100/EGM17735, an International Joint Project sponsored by the Royal Society of the U.K., the Research Grants Council of Hong Kong under Grant HKU 7031/06P, the National Natural Science Foundation of China under Grant 60804028, and the Alexander von Humboldt Foundation of Germany.

${ }^{a}$ School of Information Sciences and Technology, Donghua University, Shanghai 200051, China.

${ }^{b}$ Department of Information Systems and Computing, Brunel University, Uxbridge, Middlesex, UB8 3PH, United Kingdom.

${ }^{c}$ Department of Mechanical Engineering, The University of Hong Kong, Pokfulam Road, Hong Kong.

${ }^{d}$ UNESCO-EOLSS Joint Committee, PO Box 2623, Abu Dhabi, United Arab Emirates. The work of G. P. Rao was conducted during his research visit to Brunel University supported by the Royal Society of the U.K.

* Corresponding author. Email: Zidong. Wang@brunel.ac.uk 
the externally set control parameters $[8,18,26,33]$, and therefore state-dependent stochastic noise should be recognized as a characteristic that has to be taken into account when modeling GRNs. Furthermore, it is well known that the modeling error is unavoidable in the model identified from the measurement data. In other words, there should exist 'parameter uncertainties' in those models that are constructed from real-time data.

The complexity originated from the time delays, intrinsic fluctuation and modeling errors poses significant challenges to gene expression data analysts. A typical problem is how to obtain the steady-state values of the actual network state components (the concentrations of the mRNA and protein) based on the model identified from the measurement data. It should be pointed out that mathematical models without addressing the aforementioned inherent complexity may have wrong predictions of the mRNA and protein concentrations. Unfortunately, this issue has been largely overlooked in the literature because of the difficulty in theoretical research. It is, therefore, the main purpose of this paper to shorten such a gap by studying the so-called filtering problem, that is, predicting the network state such that the estimation error exponentially converges to zero in the mean square sense in the presence of transmission delays, parameter uncertainties and intrinsic fluctuations. Note that a similar filtering issue has been investigated in [37] for a linear stochastic GRN with variance constraints where the time delay has been ignored.

Although the filtering problem has been extensively studied in the control and signal processing communities (see [10-12,34,35] and references therein), the filtering problem for GRNs of specific structures still remains a challenging issue, on which the latest analysis techniques (e.g. careful construction of Lyapunov-Krasovskii functional and delay-dependent derivation) could be of help to provide enhanced stability conditions $[12,14,36]$. To facilitate the readers in biology area, let us briefly discuss the Lyapunov-Krasovskii functional theory, linear matrix inequality technique and exponentially mean square stability. Lyapunov's direct method (also called the second method of Lyapunov) allows us to determine the stability of a system without explicitly solving differential equations. The method is a generalization of the idea that if there is some "measure of energy" in a system, then we can study the rate of change of the energy of the system to ascertain stability [17]. In case of systems with time-delay, such measure of energy is often adopted as the Lyapunov-Krasovkii functional (LKF), which is typically of the quadratic form. By calculating the derivative of the LKF, it is usually concluded that the overall time-delay system is stable if certain linear matrix inequalities (LMIs) are feasible [12, 14,36]. Note that the solvability of LMIs can be easily checked by using the Matlab toolbox, and a growing number of dynamics analysis problems can be converted into the feasibility of LMIs [2]. The Lyapunov-Krasovkii functional theory can be easily extended to check the mean-square stability of stochastic systems [24], where the LMI framework can still be applied, see e.g. [34,35].

This paper is concerned with the filtering problem for a class of linear GRNs with state-dependent stochastic disturbances, polytopic uncertainties as well as time-varying state delays. The stochastic disturbance is described by a scalar Brownian motion and the time delays enter into both the translation process and feedback regulation process. In order to estimate the true concentrations of the mRNA and protein, we design a linear filter with guaranteed exponential convergence of the estimation error dynamics. By using the linear matrix inequality (LMI) technique and the free-weighting delay-dependent technique, sufficient conditions are derived for ensuring the exponentially mean square stability with a prescribed decay rate, and then the filter gain is characterized in terms of the solution to a set of LMIs, which can be easily solved by using available software packages. A simulation example is illustrated for a gene expression model.

Notation. Throughout this paper, $\mathbb{R}^{n}$ denotes the $n$ dimensional Euclidean space, $L_{2}[0, \infty)$ is the space of square-integrable vector functions over $[0, \infty),|\cdot|$ refers to the Euclidean norm in $\mathbb{R}^{n}$ and $\|\cdot\|_{2}$ stands for the usual $L_{2}[0, \infty)$ norm. $I$ denotes the identity matrix of compatible dimension. The notation $X \geq Y$ (respectively, $X>Y$ ), where $X$ and $Y$ are real symmetric matrices, means that $X-Y$ is positive semi-definite 
(respectively, positive definite). For a matrix $M, M^{T}$ represents its transpose and $\|M\|$ denotes its spectral norm. When $M$ is real symmetric, $\lambda_{\max }(M)$ (respectively, $\lambda_{\min }(M)$ ) stands for its maximum (respectively, minimum) eigenvalue. $\left(\Omega, \mathcal{F},\left\{\mathcal{F}_{t}\right\}_{t \in \mathbb{R}}, \mathcal{P}\right)$ is a complete probability space with a filtration $\left\{\mathcal{F}_{t}\right\}_{t \in \mathbb{R}}$ satisfying the usual conditions (i.e., the filtration contains all $P$-null sets and is right continuous). $\mathbb{E}\{x\}$ stands for the expectation of a stochastic variable $x$. The shorthand $\operatorname{diag}\left\{M_{1}, \ldots, M_{n}\right\}$ denotes a block diagonal matrix with diagonal blocks $M_{1}, \ldots, M_{n}$ and $0_{m \times n}$ denotes a $m \times n$ block matrix in which every block is 0 . In symmetric block matrices, the symbol $*$ is used as an ellipsis for terms induced by symmetry. Matrices, if the dimensions are not explicitly stated, are assumed to have compatible dimensions .

\section{Problem Formulation}

In $[6,16]$, the following linear differential equation model of genetic regulatory networks

$$
\frac{d}{d t} x(t)=M x(t)
$$

has been proposed and discussed, where $x(t) \in \mathbb{R}^{n}$ contains the mRNA and protein concentrations as a function of time $t, M=\left[M_{i j}\right]_{n \times n}$ is a constant matrix whose element $M_{i j}$ represents the effect of the concentration of mRNA or protein $j$ on the concentration of mRNA or protein $i$.

Recently, in [3], the model (1) has been generalized to account for the polytopic uncertainties and stochastic disturbances and the following uncertain stochastic genetic regulatory network model has been considered:

$$
d x(t)=N_{i} x(t) d t+M_{i} x(t) d \omega(t), \quad i=1,2, \ldots, l .
$$

Note that the model (2) can be obtained by linearizing the following kind of nonlinear stochastic genetic regulatory networks

$$
d x(t)=N(x(t)) d t+M(x(t)) d \omega(t)
$$

by using the global linearization techniques [2] in order to avoid solving a nonlinear inequality when analyzing the stability. It is worth mentioning that the robust filtering circuit design problems and $H_{\infty}$ stabilization design problems have been considered for the nonlinear genetic regulatory networks in $[4,5]$ without timedelays, and pioneering results have been obtained in the area.

As discussed in the introduction, time delays may play an important role in the dynamics of genetic networks, and mathematical models without addressing the delay effects may even have wrong predictions of the mRNA and protein concentrations [30,31]. In this paper, we extend the model (2) further by incorporating time delays in both the translation process and feedback regulation process. Specifically, we consider the following uncertain genetic regulatory networks with state-dependent stochastic disturbances and time-varying delay:

$$
\left(\Sigma_{0}\right):\left\{\begin{aligned}
d x(t) & =[A(t) x(t)+B(t) x(t-d(t))] d t+E(t) x(t) d \omega(t) \\
d y(t) & =[C(t) x(t)+D(t) x(t-d(t))] d t+F(t) x(t) d \omega(t) \\
x(t) & =\phi_{1}(t), \quad \forall t \in[-2 \tau, 0]
\end{aligned}\right.
$$

where $x(t) \in \mathbb{R}^{n}$ denotes the concentrations of mRNA and protein of the GRN, $y(t) \in \mathbb{R}^{p}$ represents the expression level of mRNA and protein of the GRN, and $\phi_{1}(t)$ is the initial function of $x(t)$. The state transition matrix $A(t) \in \mathbb{R}^{n \times n}$ shows the key information on the interactions of the mRNA and protein on each other. The matrix $C(t) \in \mathbb{R}^{p \times n}$ is the transformation matrix between the observation variables and the internal state variables of the GRN. $\omega(t)$ is a scalar Brownian motion with zero mean value and unit variance. $d(t)$ is the time-varying delay that denotes the translation delay as well as the feedback regulation delay, and satisfies

$$
0<d(t) \leq \tau<\infty \text { and } \dot{d}(t) \leq \mu<1
$$


where $\tau$ and $\mu$ are real constant scalars.

Assumption 1: The matrices $A(t), B(t), C(t), D(t), E(t)$ and $F(t)$ satisfy the following real convex polytopic uncertain constraint:

$$
\begin{aligned}
& \Omega:=[A(t) B(t) C(t) D(t) E(t) \quad F(t)] \in \mathcal{S}, \\
& \mathcal{S}:=\left\{\Omega(\lambda(t)) \mid \Omega(\lambda(t))=\sum_{j=1}^{s} \lambda_{j}(t) \Omega_{j} ; \sum_{j=1}^{s} \lambda_{j}(t)=1, \quad \lambda_{j}(t) \geq 0\right\}
\end{aligned}
$$

where $\Omega_{j}:=\left[\begin{array}{llllll}A_{j} & B_{j} & C_{j} & D_{j} & E_{j} & F_{j}\end{array}\right]$ represents the $j$ th vertex of the polytope, and $A_{j}, B_{j}, C_{j}, D_{j}, E_{j}$ and $F_{j}(j=1,2, \cdots, s)$ are all constant matrices.

Remark 1: The polytopic-type uncertainty often exists in practical systems. The issues on stability analysis and filter design for systems with polytopic uncertainties have been extensively dealt with and many results have been reported in the literature, see e.g. $[10-12,14]$. Note that many practical systems possess parameter uncertainties which can be either exactly modeled or overbounded by the polytopic uncertainty $\mathcal{S}$. Furthermore, it has been shown in [3] that the GRNs with polytopic uncertainties are an important kind of models of biological significance that is worth investigation.

Note that the system parameters of (3), though time-varying, are described by means of a polytope, and then the dynamics analysis problem for (3) could be transformed into the corresponding ones on the constant vertices. Therefore, for the sake of simplicity, we first consider the system (3) with constant parameters:

$$
(\Sigma):\left\{\begin{aligned}
d x(t) & =[A x(t)+B x(t-d(t))] d t+E x(t) d \omega(t) \\
d y(t) & =[C x(t)+D x(t-d(t))] d t+F x(t) d \omega(t) \\
x(t) & =\phi_{1}(t), \quad \forall t \in[-2 \tau, 0]
\end{aligned}\right.
$$

We will see later that the dynamics analysis results for the original system (3) can be built on those on the system (6).

Remark 2: To simplify the stability analysis issue, the equilibrium point can be shifted to the origin. In other words, in the case the concentrations of mRNA and protein do not converge to the origin when time tends to infinity, we could always make a corresponding translation of the system states.

In this paper, we endeavor to estimate the concentrations of mRNA and protein $x(t)$ through their expression level $y(t)$. The linear filter considered here is of the following form

$$
\left(\Sigma_{f}\right):\left\{\begin{aligned}
d \hat{x}(t) & =\hat{A} \hat{x}(t) d t+\hat{B} d y(t) \\
\hat{x}(t) & =\phi_{2}(t), \quad \forall t \in[-2 \tau, 0]
\end{aligned}\right.
$$

where $\hat{x}(t) \in \mathbb{R}^{n}$ is the estimate for $x(t), \phi_{2}(t)$ is the initial function of $\hat{x}(t)$, and $\hat{A}$ and $\hat{B}$ are filter parameters to be determined.

Defining

$$
\bar{x}(t):=\left[\begin{array}{l}
x(t) \\
\hat{x}(t)
\end{array}\right], \quad \bar{x}(t-d(t)):=\left[\begin{array}{l}
x(t-d(t)) \\
\hat{x}(t-d(t))
\end{array}\right],
$$

and augmenting $(\Sigma)$ and $\left(\Sigma_{f}\right)$, the state estimation dynamics is governed by the following system:

$$
\left(\Sigma_{e}\right):\left\{\begin{aligned}
d \bar{x}(t) & =[\bar{A} \bar{x}(t)+\bar{B} Z \bar{x}(t-d(t))] d t+\bar{E} Z \bar{x}(t) d \omega(t), \\
\bar{x}(t) & =\phi(t), \quad \forall t \in[-2 \tau, 0],
\end{aligned}\right.
$$

where

$$
\bar{A}=\left[\begin{array}{cc}
A & 0 \\
\hat{B} C & \hat{A}
\end{array}\right], \bar{B}=\left[\begin{array}{c}
B \\
\hat{B} D
\end{array}\right], \bar{E}=\left[\begin{array}{c}
E \\
\hat{B} F
\end{array}\right], Z=\left[\begin{array}{ll}
I & 0
\end{array}\right], \phi(t)=\left[\begin{array}{c}
\phi_{1}(t) \\
\phi_{2}(t)
\end{array}\right]
$$


For presentation convenience, we set

$$
\xi(t)=\bar{A} \bar{x}(t)+\bar{B} Z \bar{x}(t-d(t))
$$

and then $\left(\Sigma_{e}\right)$ in $(8)$ can be rewritten as

$$
d \bar{x}(t)=\xi(t) d t+\bar{E} Z \bar{x}(t) d \omega(t) .
$$

Before formulating the problem to be investigated, we introduce the following stability concept for the augmented system (8).

Definition 1: System (8) is said to be exponentially mean square stable if there exist scalars $\alpha>0$ and $\beta>0$ such that

$$
\mathbb{E}|\bar{x}(t, \phi)|^{2} \leq \alpha e^{-\beta t} \sup _{-2 \tau \leq t \leq 0} \mathbb{E}|\phi(t)|^{2}, \quad \forall \phi(t) \in \mathbb{R}^{2 n \times n}
$$

or, equivalently,

$$
\lim _{t \rightarrow \infty} \sup \frac{1}{t} \log \left(\mathbb{E}|\bar{x}(t, \phi)|^{2}\right) \leq-\beta,
$$

where $\phi(t):=\left[\begin{array}{ll}\phi_{1}^{T}(t) & \phi_{2}^{T}(t)\end{array}\right]^{T}$ is the initial function of $\bar{x}(t)$ on the interval $[-2 \tau, 0]$ and $\beta$ is the exponential decay rate.

In this paper, our goal is to design a linear filter of the form $(7)$ for the system $(\Sigma)$ in $(6)$ such that, for all admissible time-varying delays, polytopic uncertainties and stochastic disturbances, the augmented system (8) that governs the state estimation dynamics is exponentially mean square stable.

\section{MAIN RESUlts}

In this section, we shall deal with the robust filtering problem for the augmented system (8) by using a combination of delay-dependent synthesis approach, Lyapunov-Krasovskii functional theory and Itô differential formula.

\section{A. Filter analysis}

First, let the filter parameter be given and we study the filtering analysis problem. The following lemma will be used in the proof of our main results in this paper.

Lemma 1: [2] (Schur Complement) Given constant matrices $\Sigma_{1}, \Sigma_{2}, \Sigma_{3}$ where $\Sigma_{1}=\Sigma_{1}^{T}$ and $0<\Sigma_{2}=\Sigma_{2}^{T}$. Then $\Sigma_{1}+\Sigma_{3}^{T} \Sigma_{2}^{-1} \Sigma_{3}<0$ if and only if

$$
\left[\begin{array}{cc}
\Sigma_{1} & \Sigma_{3}^{T} \\
\Sigma_{3} & -\Sigma_{2}
\end{array}\right]<0 \quad \text { or } \quad\left[\begin{array}{cc}
-\Sigma_{2} & \Sigma_{3} \\
\Sigma_{3}^{T} & \Sigma_{1}
\end{array}\right]<0 .
$$

For simplicity of presentation, we first consider the stochastic time-delay GRN (6) without uncertainties, but eventually we will extend our main result to the case when the GRN involves polytopic uncertainties. In the following theorem, a delay-dependent LMI method is developed to obtain sufficient conditions ensuring the solvability of the filtering problem.

Theorem 1: Consider system (8) with given filter parameters. For a prescribed constant $\beta>0$, if there exist positive definite matrices $P>0, Q_{i}>0, R_{i}>0, Z_{l}>0$ and matrices $S_{l}=\left[\begin{array}{lll}S_{l 1}^{T} Z & S_{l 2}^{T} & S_{l 3}^{T}\end{array}\right]^{T}$ 
$(i=1,2 ; l=1,2,3)$ such that the following linear matrix inequalities

$$
\begin{aligned}
& {\left[\begin{array}{ccc}
\Xi & \tau S_{3} & \tau S_{1} \\
* & -\tau Z_{3} & 0 \\
* & 0 & -\tau Z_{1}
\end{array}\right]<0,} \\
& {\left[\begin{array}{ccc}
\Xi & \tau S_{3} & \tau S_{2} \\
* & -\tau Z_{3} & 0 \\
* & 0 & -\tau Z_{2}
\end{array}\right]<0,} \\
& Z_{1}<R_{1}, \quad Z_{2}<R_{1}, \quad Z_{3}<R_{2}
\end{aligned}
$$

hold, where

$$
\begin{aligned}
\Xi & :=\Xi_{1}+\Xi_{2}+\Xi_{2}^{T}, \quad \Xi_{1}:=\left[\begin{array}{ccc}
\Xi_{11} & P \bar{B}+\frac{e^{\beta \tau}-1}{\beta} \bar{A}^{T} Z^{T}\left(R_{1}+R_{2}\right) Z \bar{B} & 0 \\
* & -(1-\mu) Q_{1}+\frac{e^{\beta \tau}-1}{\beta} \bar{B}^{T} Z^{T}\left(R_{1}+R_{2}\right) Z \bar{B} & 0 \\
* & * & -Q_{2}
\end{array}\right], \\
\Xi_{2} & :=\left[S_{1} Z+S_{3} Z-S_{1}+S_{2}-S_{2}-S_{3}\right], \\
\Xi_{11} & :=\beta P+P \bar{A}+\bar{A}^{T} P+Z^{T} \bar{E}^{T} P \bar{E} Z+e^{\beta \tau} Z^{T}\left(Q_{1}+Q_{2}\right) Z+\frac{e^{\beta \tau}-1}{\beta} \bar{A}^{T} Z^{T}\left(R_{1}+R_{2}\right) Z \bar{A},
\end{aligned}
$$

then the augmented system (8) is exponentially mean square stable.

Proof: To prove the exponential mean square stability of (8) under condition (12)-(14), we consider the following Lyapunov-Krasovskii functional candidate:

$$
\begin{aligned}
V(t)= & e^{\beta t} \bar{x}^{T}(t) P \bar{x}(t)+\int_{t-d(t)}^{t} e^{\beta(s+\tau)} \bar{x}^{T}(s) Z^{T} Q_{1} Z \bar{x}(s) d s+\int_{t-\tau}^{t} e^{\beta(s+\tau)} \bar{x}^{T}(s) Z^{T} Q_{2} Z \bar{x}(s) d s \\
& +\int_{-\tau}^{0} \int_{t+s}^{t} e^{\beta(\theta-s)} \xi^{T}(\theta) Z^{T}\left(R_{1}+R_{2}\right) Z \xi(\theta) d \theta d s .
\end{aligned}
$$

It follows from Itô's differential formula [21] that

$$
d V(t)=\mathcal{L} V(t) d t+2 e^{\beta t} \bar{x}^{T}(t) P \bar{E} Z \bar{x}(t) d \omega(t),
$$

where

$$
\begin{aligned}
\mathcal{L} V(t)= & \beta e^{\beta t} \bar{x}^{T}(t) P \bar{x}(t)+2 e^{\beta t} \bar{x}^{T}(t) P[\bar{A} \bar{x}+\bar{B} Z \bar{x}(t-d(t))]+e^{\beta t} \bar{x}^{T}(t) Z^{T} \bar{E}^{T} P \bar{E} Z \bar{x}(t) \\
& +e^{\beta(t+\tau)} \bar{x}^{T}(t) Z^{T} Q_{1} Z \bar{x}(t)-(1-\dot{d}(t)) e^{\beta(t-d(t)+\tau)} \bar{x}^{T}(t-d(t)) Z^{T} Q_{1} Z \bar{x}(t-d(t)) \\
& +e^{\beta(t+\tau)} \bar{x}^{T}(t) Z^{T} Q_{2} Z \bar{x}(t)-e^{\beta t} \bar{x}^{T}(t-\tau) Z^{T} Q_{2} Z \bar{x}(t-\tau) \\
& +e^{\beta t} \frac{e^{\beta \tau}-1}{\beta} \xi^{T}(t) Z^{T}\left(R_{1}+R_{2}\right) Z \xi(t)-e^{\beta t} \int_{t-d(t)}^{t} \xi^{T}(s) Z^{T} R_{1} Z \xi(s) d s \\
& -e^{\beta t} \int_{t-\tau}^{t-d(t)} \xi^{T}(s) Z^{T} R_{1} Z \xi(s) d s-e^{\beta t} \int_{t-\tau}^{t} \xi^{T}(s) Z^{T} R_{2} Z \xi(s) d s .
\end{aligned}
$$

By the Newton-Leibniz formula, for any appropriately dimension matrices $S_{1}, S_{2}, S_{3}$, the following equalities

$$
\begin{aligned}
& \eta^{T}(t) S_{1} Z\left[\bar{x}(t)-\bar{x}(t-d(t))-\int_{t-d(t)}^{t} d \bar{x}(t)\right]=0 \\
& \eta^{T}(t) S_{2} Z\left[\bar{x}(t-d(t))-\bar{x}(t-\tau)-\int_{t-\tau}^{t-d(t)} d \bar{x}(t)\right]=0 \\
& \eta^{T}(t) S_{3} Z\left[\bar{x}(t)-\bar{x}(t-\tau)-\int_{t-\tau}^{t} d \bar{x}(t)\right]=0
\end{aligned}
$$


are true, where $\eta(t)=\left[\begin{array}{lll}\bar{x}^{T}(t) & \bar{x}^{T}(t-d(t)) Z^{T} \quad \bar{x}^{T}(t-\tau) Z^{T}\end{array}\right]^{T}$.

Noticing the relationship between the time-varying delay $d(t)$ and the constants $\tau, \mu$, and using the notation of $\xi(t)$, we have

$$
\begin{aligned}
\mathcal{L} V(t) \leq & e^{\beta t}\left\{\beta \bar{x}^{T}(t) P \bar{x}(t)+2 \bar{x}^{T}(t) P[\bar{A} \bar{x}+\bar{B} Z \bar{x}(t-d(t))]+\bar{x}^{T}(t) Z^{T} \bar{E}^{T} P \bar{E} Z \bar{x}(t)\right. \\
& +e^{\beta \tau} \bar{x}^{T}(t) Z^{T} Q_{1} Z \bar{x}(t)-(1-\mu) \bar{x}^{T}(t-d(t)) Z^{T} Q_{1} Z \bar{x}(t-d(t)) \\
& +e^{\beta \tau} \bar{x}^{T}(t) Z^{T} Q_{2} Z \bar{x}(t)-\bar{x}^{T}(t-\tau) Z^{T} Q_{2} Z \bar{x}(t-\tau) \\
& +\frac{e^{\beta \tau}-1}{\beta} \xi^{T}(t) Z^{T}\left(R_{1}+R_{2}\right) Z \xi(t)-\int_{t-d(t)}^{t} \xi^{T}(s) Z^{T} R_{1} Z \xi(s) d s \\
& -\int_{t-\tau}^{t-d(t)} \xi^{T}(s) Z^{T} R_{1} Z \xi(s) d s-\int_{t-\tau}^{t} \xi^{T}(s) Z^{T} R_{2} Z \xi(s) d s \\
& +2 \eta^{T}(t) S_{1} Z\left[\bar{x}(t)-\bar{x}(t-d(t))-\int_{t-d(t)}^{t} \xi(s) d s-\int_{t-d(t)}^{t} \bar{E} Z \bar{x}(s) d \omega(s)\right] \\
& +2 \eta^{T}(t) S_{2} Z\left[\bar{x}(t-d(t))-\bar{x}(t-\tau)-\int_{t-\tau}^{t-d(t)} \xi(s) d s-\int_{t-\tau}^{t-d(t)} \bar{E} Z \bar{x}(s) d \omega(s)\right] \\
& +2 \eta^{T}(t) S_{3} Z\left[\bar{x}(t)-\bar{x}(t-\tau)-\int_{t-\tau}^{t} \xi(s) d s-\int_{t-\tau}^{t} \bar{E} Z \bar{x}(s) d \omega(s)\right] \\
& +d(t) \eta^{T}(t) S_{1} Z_{1}^{-1} S_{1}^{T} \eta(t)-\int_{t-d(t)}^{t} \eta^{T}(t) S_{1} Z_{1}^{-1} S_{1}^{T} \eta(t) d s \\
& +(\tau-d(t)) \eta^{T}(t) S_{2} Z_{2}^{-1} S_{2}^{T} \eta(t)-\int_{t-\tau}^{t-d(t)} \eta^{T}(t) S_{2} Z_{2}^{-1} S_{2}^{T} \eta(t) d s \\
& +\tau \eta^{T}(t) S_{3} Z_{3}^{-1} S_{3}^{T} \eta(t)-\int_{t-\tau}^{t} \eta^{T}(t) S_{3} Z_{3}^{-1} S_{3}^{T} \eta(t) d s .
\end{aligned}
$$

It follows from (21) and the conditions of $Z_{1}<R_{1}, Z_{2}<R_{1}, Z_{3}<R_{2}$ in (14) that

$$
\begin{aligned}
\mathbb{E}\{\mathcal{L} V(t)\} \leq & e^{\beta t} \mathbb{E}\left\{\Gamma+d(t) \eta^{T}(t) S_{1} Z_{1}^{-1} S_{1}^{T} \eta(t)+(\tau-d(t)) \eta^{T}(t) S_{2} Z_{2}^{-1} S_{2}^{T} \eta(t)+\tau \eta^{T}(t) S_{3} Z_{3}^{-1} S_{3}^{T} \eta(t)\right. \\
& -\int_{t-d(t)}^{t}\left[\eta^{T}(t) S_{1}+\xi^{T}(s) Z^{T} R_{1}\right] R_{1}^{-1}\left[S_{1} \eta(t)+R_{1} Z \xi(s)\right] d s \\
& -\int_{t-\tau}^{t-d(t)}\left[\eta^{T}(t) S_{2}+\xi^{T}(s) Z^{T} R_{1}\right] R_{1}^{-1}\left[S_{2} \eta(t)+R_{1} Z \xi(s)\right] d s \\
& -\int_{t-\tau}^{t}\left[\eta^{T}(t) S_{3}+\xi^{T}(s) Z^{T} R_{2}\right] R_{2}^{-1}\left[S_{3} \eta(t)+R_{2} Z \xi(s)\right] d s \\
& \left.-2 \eta^{T}(t)\left[\int_{t-d(t)}^{t} S_{1} E x(s) d \omega(s)+\int_{t-\tau}^{t-d(t)} S_{2} E x(s) d \omega(s)+\int_{t-\tau}^{t} S_{3} E x(s) d \omega(s)\right]\right\}
\end{aligned}
$$

where

$$
\begin{aligned}
\Gamma= & \beta \bar{x}^{T}(t) P \bar{x}(t)+2 \bar{x}^{T}(t) P[\bar{A} \bar{x}+\bar{B} Z \bar{x}(t-d(t))]+\bar{x}^{T}(t) Z^{T} \bar{E}^{T} P \bar{E} Z \bar{x}(t)+e^{\beta \tau} \bar{x}^{T}(t) Z^{T} Q_{1} Z \bar{x}(t) \\
& -(1-\mu) \bar{x}^{T}(t-d(t)) Z^{T} Q_{1} Z \bar{x}(t-d(t))+e^{\beta \tau} \bar{x}^{T}(t) Z^{T} Q_{2} Z \bar{x}(t)-\bar{x}^{T}(t-\tau) Z^{T} Q_{2} Z \bar{x}(t-\tau) \\
& +\frac{e^{\beta \tau}-1}{\beta}[\bar{A} \bar{x}(t)+\bar{B} Z \bar{x}(t-d(t))]^{T} Z^{T}\left(R_{1}+R_{2}\right) Z[\bar{A} \bar{x}+\bar{B} Z \bar{x}(t-d(t))] \\
& +2 \eta^{T}(t) S_{1} Z[\bar{x}(t)-\bar{x}(t-d(t))]+2 \eta^{T}(t) S_{2} Z[\bar{x}(t-d(t))-\bar{x}(t-\tau)] \\
& +2 \eta^{T}(t) S_{3} Z[\bar{x}(t)-\bar{x}(t-\tau)] .
\end{aligned}
$$


Then, our next goal is to prove that

$$
\Gamma+\tau \eta^{T}(t) S_{3} Z_{3}^{-1} S_{3}^{T} \eta(t)+d(t) \eta^{T}(t) S_{1} Z_{1}^{-1} S_{1}^{T} \eta(t)+(\tau-d(t)) \eta^{T}(t) S_{2} Z_{2}^{-1} S_{2}^{T} \eta(t)<0
$$

which is equivalent to

$$
\begin{gathered}
\Gamma+\tau \eta^{T}(t) S_{3} Z_{3}^{-1} S_{3}^{T} \eta(t)+\tau \eta^{T}(t) S_{1} Z_{1}^{-1} S_{1}^{T} \eta(t)<0 \\
\Gamma+\tau \eta^{T}(t) S_{3} Z_{3}^{-1} S_{3}^{T} \eta(t)+\tau \eta^{T}(t) S_{2} Z_{2}^{-1} S_{2}^{T} \eta(t)<0
\end{gathered}
$$

which would hold if we have

$$
\Xi+\tau S_{3} Z_{3}^{-1} S_{3}^{T}+\tau S_{1} Z_{1}^{-1} S_{1}^{T}<0, \quad \Xi+\tau S_{3} Z_{3}^{-1} S_{3}^{T}+\tau S_{2} Z_{2}^{-1} S_{2}^{T}<0
$$

By Schur complement, we can obtain from (12) and (13) that (27) is true, hence $\mathbb{E}\{\mathcal{L} V(t)\}<0$.

The exponentially mean square stability of system (8) can be proved as follows. From the definitions of $\xi(t), \bar{x}(t), \phi(t)$ and (15), there exists a positive scalar $\delta$ such that

$$
\mathbb{E} V(t) \geq e^{\beta t} \lambda_{\min }(P) \mathbb{E}|\bar{x}(t)|^{2}
$$

and

$$
\begin{aligned}
\mathbb{E} V(0) & \leq\left\{\|P\|+\tau e^{\beta \tau}\left[\left\|Q_{1}\right\|+\left\|Q_{2}\right\|+2 \tau\left(\left\|R_{1}\right\|+\left\|R_{2}\right\|\right)\left(\|\bar{A}\|^{2}+\|\bar{B}\|^{2}\right)\right]\right\} \sup _{-2 \tau \leq t \leq 0}|\phi(t)|^{2} \\
& \leq \delta \sup _{-2 \tau \leq t \leq 0} \mathbb{E}|\phi(t)|^{2} .
\end{aligned}
$$

By Itô's formula [21], we obtain that

$$
\mathbb{E} V(t)=\mathbb{E} V(0)+\mathbb{E} \int_{0}^{t} \mathcal{L} V(s) d s \leq \delta \sup _{-2 \tau \leq t \leq 0} \mathbb{E}|\phi(t)|^{2}
$$

It follows from (28), (29) and (30) that

$$
\mathbb{E}|\bar{x}(t, \phi)|^{2} \leq \frac{\delta}{\lambda_{\min }(P)} e^{-\beta t} \sup _{-2 \tau \leq t \leq 0} \mathbb{E}|\phi(t)|^{2}
$$

or, equivalently,

$$
\lim _{t \rightarrow \infty} \sup \frac{1}{t} \log \left(\mathbb{E}|\bar{x}(t, \phi)|^{2}\right) \leq-\beta
$$

which indicates that the trivial solution of (8) is exponentially mean square stable and the exponential decay rate is $\beta$. The proof is now complete.

Remark 3: In Theorem 1, the decay rate $\beta$ is included that serves as an important index characterizing the transient behavior of the filtering error dynamics, i.e., how fast the error dynamics converges to zero. Note that the parameter $\beta$ is adjustable according to practical requirements. A similar approach to characterizing the decay rate has been exploited in [29] for a class of stochastic nonlinear neural networks with time delay.

\section{B. Filter synthesis}

The desired filter parameters can be determined in the following theorem by solving a set of LMIs.

Theorem 2: Consider system (8) without uncertainties. For a prescribed constant $\beta>0$, if there exist positive definite matrices $X>0, \mathcal{Y}>0 Q_{i}>0, R_{i}>0, Z_{l}$ and matrices $S_{l k},(i=1,2 ; l=1,2,3 ; k=1,2,3)$ 
such that the following linear matrix inequalities

$$
\begin{aligned}
& {\left[\begin{array}{cc}
\Pi & \bar{S}_{1} \\
\bar{S}_{1}^{T} & -\tau Z_{1}
\end{array}\right]+\Phi+\Phi^{T}<0,} \\
& {\left[\begin{array}{cc}
\Pi & \bar{S}_{2} \\
\bar{S}_{2}^{T} & -\tau Z_{2}
\end{array}\right]+\Phi+\Phi^{T}<0,} \\
& Z_{1}<R_{1}, \quad Z_{2}<R_{1}, \quad Z_{3}<R_{2},
\end{aligned}
$$

hold, where

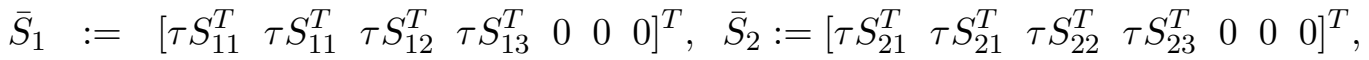

$$
\begin{aligned}
& \Pi:=\left[\begin{array}{ccccccc}
\Pi_{1} & \Pi_{2} & \mathcal{Y} B & 0 & \Pi_{4} & A^{T}\left(R_{1}+R_{2}\right) & \tau S_{31} \\
* & \Pi_{3} & X B+\tilde{B} D & 0 & \Pi_{5} & A^{T}\left(R_{1}+R_{2}\right) & \tau S_{31} \\
* & * & -(1-\mu) Q_{1} & 0 & 0 & B^{T}\left(R_{1}+R_{2}\right) & \tau S_{32} \\
* & * & * & -Q_{2} & 0 & 0 & \tau S_{33} \\
* & * & * & * & -\Lambda & 0 & 0 \\
* & * & * & * & * & -\frac{\beta}{e^{\beta \tau}-1}\left(R_{1}+R_{2}\right) & 0 \\
* & * & * & * & * & * & -\tau Z_{3}
\end{array}\right],
\end{aligned}
$$

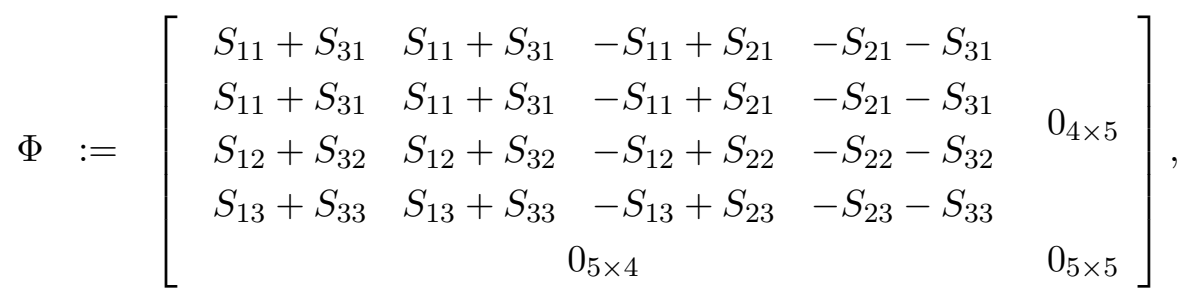

$$
\begin{aligned}
& \Pi_{1}:=A^{T} \mathcal{Y}+\mathcal{Y} A+e^{\beta \tau}\left(Q_{1}+Q_{2}\right)+\beta \mathcal{Y}, \Pi_{2}:=A^{T} X+\mathcal{Y} A+C^{T} \tilde{B}^{T}+\tilde{A}^{T}+e^{\beta \tau}\left(Q_{1}+Q_{2}\right)+\beta \mathcal{Y}, \\
& \Pi_{3}:=A^{T} X+X A+\tilde{B} C+C^{T} \tilde{B}^{T}+e^{\beta \tau}\left(Q_{1}+Q_{2}\right)+\beta X, \Pi_{4}:=\left[E^{T} \mathcal{Y} E^{T} X+F^{T} \tilde{B}^{T}\right], \\
& \Pi_{5}:=\left[\begin{array}{ll}
E^{T} \mathcal{Y} & E^{T} X+F^{T} \tilde{B}^{T}
\end{array}\right], \Lambda:=\left[\begin{array}{cc}
\mathcal{Y} & \mathcal{Y} \\
\mathcal{Y} & X
\end{array}\right],
\end{aligned}
$$

with $0_{n_{1} \times n_{2}}$ being a $n_{1} \times n_{2}$ zero block-matrix whose dimension is compatible with other block entries in $\Phi$, then system (8) is exponentially mean square stable. In this case, the parameters of the desired filter $\left(\Sigma_{f}\right)$ are given as follows:

$$
\hat{A}:=(\mathcal{Y}-X)^{-1} \tilde{A}, \hat{B}:=(\mathcal{Y}-X)^{-1} \tilde{B}
$$

Proof: Define

$$
P=\left[\begin{array}{cc}
X & \mathcal{Y}-X \\
\mathcal{Y}-X & X-\mathcal{Y}
\end{array}\right]>0, \quad \Upsilon=\left[\begin{array}{cc}
Y & I \\
Y & 0
\end{array}\right]
$$

where $Y=\mathcal{Y}^{-1}>0$.

Pre- and post-multiplying the LMIs in (31) and (32) by $\operatorname{diag}\{\operatorname{diag}\{Y, I, I, I, \operatorname{diag}\{Y, I\}, I, I\}, I\}$ and its transpose, we have

$$
\begin{gathered}
{\left[\begin{array}{cc}
\bar{\Pi} & \breve{S}_{1} \\
\breve{S}_{1}^{T} & -\tau Z_{1}
\end{array}\right]+\bar{\Phi}+\bar{\Phi}^{T}<0, \quad\left[\begin{array}{cc}
\bar{\Pi} & \breve{S}_{2} \\
\breve{S}_{2}^{T} & -\tau Z_{2}
\end{array}\right]+\bar{\Phi}+\bar{\Phi}^{T}<0,} \\
Z_{1}<R_{1}, \quad Z_{2}<R_{1}, \quad Z_{3}<R_{2}
\end{gathered}
$$


where

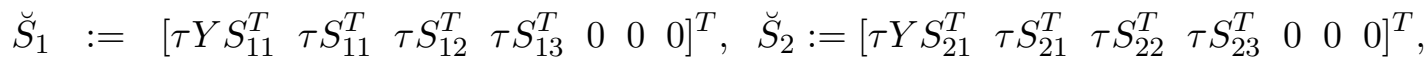

$$
\begin{aligned}
& \bar{\Pi}:=\left[\begin{array}{ccccccc}
\bar{\Pi}_{1} & \bar{\Pi}_{2} & B & 0 & \bar{\Pi}_{4} & Y A^{T}\left(R_{1}+R_{2}\right) & \tau Y S_{31} \\
* & \Pi_{3} & X B+\tilde{B} D & 0 & \bar{\Pi}_{5} & A^{T}\left(R_{1}+R_{2}\right) & \tau S_{31} \\
* & * & -(1-\mu) Q_{1} & 0 & 0 & B^{T}\left(R_{1}+R_{2}\right) & \tau S_{32} \\
* & * & * & -Q_{2} & 0 & 0 & \tau S_{33} \\
* & * & * & * & -\bar{\Lambda} & 0 & 0 \\
* & * & * & * & * & -\frac{\beta}{e^{\beta \tau}-1}\left(R_{1}+R_{2}\right) & 0 \\
* & * & * & * & * & * & -\tau Z_{3}
\end{array}\right],
\end{aligned}
$$

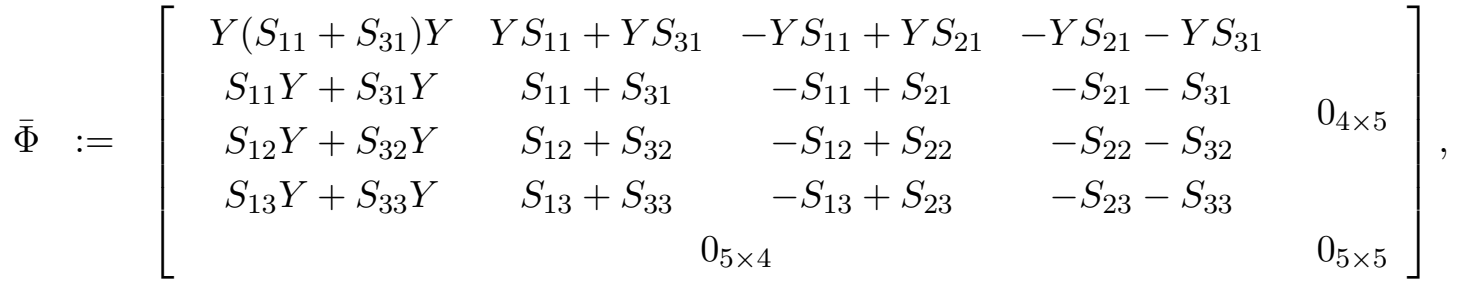

$$
\begin{aligned}
& \bar{\Pi}_{1}:=Y A^{T}+A Y+e^{\beta \tau} Y\left(Q_{1}+Q_{2}\right) Y+\beta Y \text {, } \\
& \bar{\Pi}_{2}:=Y A^{T} X+A+Y C^{T} \tilde{B}^{T}+Y \tilde{A}^{T}+e^{\beta \tau} Y\left(Q_{1}+Q_{2}\right)+\beta I \text {, } \\
& \bar{\Pi}_{4}:=\left[\begin{array}{lll}
Y E^{T} & Y E^{T} X+Y F^{T} \tilde{B}^{T}
\end{array}\right], \quad \bar{\Pi}_{5}:=\left[\begin{array}{ll}
E^{T} & E^{T} X+F^{T} \tilde{B}^{T}
\end{array}\right], \bar{\Lambda}:=\left[\begin{array}{cc}
Y & I \\
I & X
\end{array}\right] .
\end{aligned}
$$

It can be seen from the definitions of $P$ and $\Upsilon$ that the LMI in (37) is equivalent to

$$
\left[\begin{array}{ccc}
\bar{\Xi} & \tau \tilde{S}_{3} & \tau \tilde{S}_{1} \\
\tau \tilde{S}_{3}^{T} & -\tau Z_{3} & 0 \\
\tau \tilde{S}_{1}^{T} & 0 & -\tau Z_{1}
\end{array}\right]<0, \quad\left[\begin{array}{ccc}
\bar{\Xi} & \tau \tilde{S}_{3} & \tau \tilde{S}_{2} \\
\tau \tilde{S}_{3}^{T} & -\tau Z_{3} & 0 \\
\tau \tilde{S}_{2}^{T} & 0 & -\tau Z_{2}
\end{array}\right]<0,
$$

where

$$
\begin{aligned}
& \bar{\Xi}:=\bar{\Xi}_{1}+\bar{\Xi}_{2}+\bar{\Xi}_{2}^{T}, \bar{\Xi}_{1}:=\left[\begin{array}{ccccc}
\bar{\Xi}_{11} & \Upsilon^{T} P \bar{B} & 0 & \Upsilon^{T} Z^{T} \bar{E}^{T} P \Upsilon & \Upsilon^{T} \bar{A}^{T} Z^{T}\left(R_{1}+R_{2}\right) \\
* & -(1-\mu) Q_{1} & 0 & 0 & \bar{B}^{T} Z^{T}\left(R_{1}+R_{2}\right) \\
* & * & -Q_{2} & 0 & 0 \\
* & * & * & -\Upsilon^{T} P \Upsilon & 0 \\
* & * & * & * & -\frac{\beta}{e^{\beta \tau}-1}\left(R_{1}+R_{2}\right)
\end{array}\right] \text {, } \\
& \bar{\Xi}_{2}:=\left[\tilde{S}_{1} Z \Upsilon+\tilde{S}_{3} Z \Upsilon-\tilde{S}_{1}+\tilde{S}_{2}-\tilde{S}_{2}-\tilde{S}_{3}\right], \bar{\Xi}_{11}:=\Upsilon^{T}\left(\beta P+P \bar{A}+\bar{A}^{T} P+e^{\beta \tau} Z^{T}\left(Q_{1}+Q_{2}\right) Z\right) \Upsilon \text {, } \\
& \tilde{S}_{l}:=\left[\begin{array}{lllll}
S_{l 1}^{T} Z \Upsilon & S_{l 2}^{T} & S_{l 3}^{T} & 0 & 0
\end{array}\right]^{T},(l=1,2,3) .
\end{aligned}
$$

Finally, pre- and post-multiplying LMIs in (40) by $\operatorname{diag}\left\{\operatorname{diag}\left\{\Upsilon^{-T}, I, I, \Upsilon^{-T}, I\right\}, I, I\right\}$ and its transpose, we can obtain from Theorem 1 and Schur complement that system (8) is exponentially mean square stable with the given filter parameters in (35).

\section{The solution}

In the following theorem, the desired filter synthesis problem is solved for the polytopic uncertain stochastic genetic regulatory network (6) with time-varying delay. The proof of this theorem can be obtained along the similar line of that of Theorem 2, and is therefore omitted here to avoid unnecessary duplication.

Theorem 3: Consider system (8) with polytopic uncertainties satisfying Assumption 1. For a prescribed constant $\beta>0$, if there exist positive definite matrices $X>0, \mathcal{Y}>0, Q_{i}>0, R_{i}>0, Z_{l}$, and $S_{l k}>0$, 
$(i=1,2 ; l=1,2,3 ; k=1,2,3)$ such that the linear matrix inequalities (31)-(33) hold for $j=1, \cdots, s$, where the system matrices $A, B, C, D, E, F$ are substituted by $A_{j}, B_{j}, C_{j}, D_{j}, E_{j}$, $F_{j}$, then the stochastic genetic regulatory networks with polytopic uncertainties satisfying (5) and time-varying delays satisfying (4) is exponentially mean square stable and the desired filter parameters $\hat{A}, \hat{B}$ can be derived according to (35).

\section{An Illustrative Example}

In this section, a simulation example is presented to illustrate the usefulness and flexibility of the filter design method developed in this paper.

As discussed in the Section II, sometimes a linear differential equation model could be used to approximate genetic regulatory networks $[6,16]$. On the other hand, nonlinear stochastic genetic regulatory networks could be linearized by using the global linearization techniques [2] with the hope to avoid solving a nonlinear inequality when analyzing the stability [3]. Note that a linear GRN model with polytopic uncertainties and stochastic disturbances has recently been investigated in [3]. Motivated by this, we consider the following linear uncertain stochastic time-delay GRN which takes into account the time delays, polytopic uncertainty and stochastic disturbance:

$$
\left\{\begin{aligned}
d x_{1}(t) & =\left[-3 x_{1}(t)+(0.42+0.3 \rho) x_{2}(t)-0.1 x_{1}(t-d(t))+(0.12+0.1 \sigma) x_{2}(t-d(t))\right] d t+0.4 x_{1}(t) d \omega(t) \\
d x_{2}(t) & =\left[(-2.5+0.3 \rho) x_{2}(t)-0.15 x_{3}(t)-0.08 x_{2}(t-d(t))-0.14 x_{3}(t-d(t))\right] d t+0.4 x_{2}(t) d \omega(t) \\
d x_{3}(t) & =\left[0.1 x_{1}(t)-2.4 x_{3}(t)+0.1 x_{4}(t)-0.11 x_{3}(t-d(t))+0.12 x_{4}(t-d(t))\right] d t+0.4 x_{3}(t) d \omega(t) \\
d x_{4}(t) & =\left[(-2.1+0.1 \rho) x_{4}(t)+0.1 x_{5}(t)-0.21 x_{4}(t-d(t))+0.13 x_{5}(t-d(t))\right] d t+0.4 x_{4}(t) d \omega(t) \\
d x_{5}(t) & =\left[-0.2 x_{1}(t)-0.15 x_{4}(t)-2 x_{5}(t)-0.08 x_{1}(t-d(t))-0.15 x_{5}(t-d(t))\right] d t+0.4 x_{1}(t) d \omega(t)
\end{aligned}\right.
$$

where the time delay $d(t)=0.6+0.3 \sin (2 t)$, the delay bound $\tau=1$, the delay rate $\mu=0.6$, the exponential decay rate $\beta=1.0$ and

$$
C=D=0.16 I, \quad F=0.4 I
$$

where $|\rho| \leq 1,|\sigma| \leq 1$.

According to Theorem 2, the filter parameters can be calculated as follows:

$$
\begin{gathered}
\hat{A}=\left[\begin{array}{ccccc}
-7.0556 & 0.7856 & 0.0802 & 0.0006 & -0.1613 \\
0.3117 & -5.7085 & -0.2743 & 0.0006 & 0.0020 \\
0.1826 & -0.1218 & -5.5059 & 0.1806 & 0.0026 \\
0.0006 & 0.0007 & 0.0802 & -4.8326 & 0.0841 \\
-0.3579 & 0.0056 & 0.0030 & -0.1662 & -4.5953
\end{array}\right], \\
\hat{B}=\left[\begin{array}{ccccc}
2.7845 & -0.1596 & -0.0176 & 0.0019 & 0.0314 \\
-0.1488 & 2.5626 & 0.0149 & -0.0001 & 0.0027 \\
-0.0202 & 0.0248 & 2.5891 & -0.0079 & 0.0012 \\
0.0010 & 0.0002 & -0.0177 & 2.4550 & 0.0218 \\
0.0253 & -0.0035 & 0.0009 & 0.0108 & 2.4340
\end{array}\right] .
\end{gathered}
$$

Figs. 1-2 give the simulation results for the performance of the designed filter. Fig. 3 plots the actual decay rate of augmented system's state $\bar{x}(t)$ via $-\sup \frac{1}{t} \log \left(\mathbb{E}|\bar{x}(t, \rho)|^{2}\right)$ and the decay rate estimation $\beta$, where the initial condition is set to be $\phi(t)=\left[\begin{array}{llllllllll}0.1 & 0.15 & 0.3 & 0.4 & 0.5 & 0 & 0.12 & 0.25 & 0.31 & 0.45\end{array}\right]^{T}(-2 \tau \leq t \leq 0)$. It is confirmed from the simulation results that the expected exponentially mean square stability has been guaranteed. 


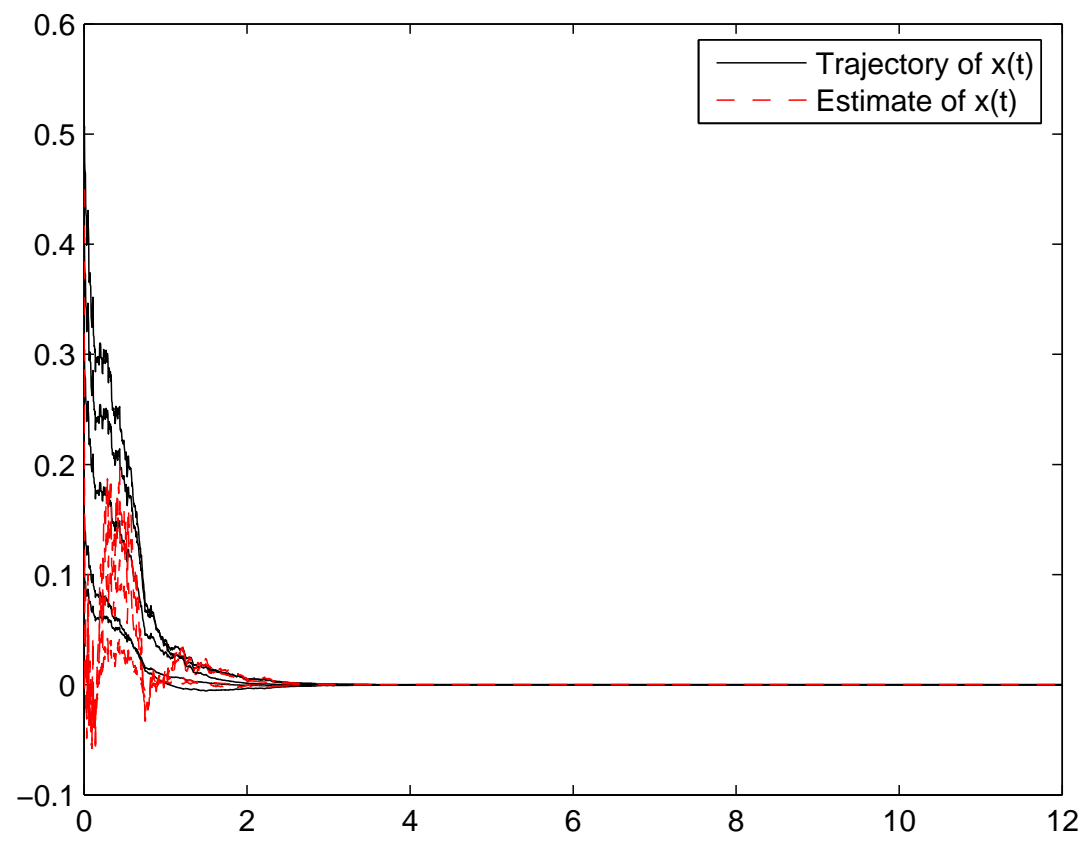

Fig. 1. Trajectory and estimate of $x(t)$

\section{Conclusions}

In this paper, we have investigated the filtering problem on a class of stochastic genetic regulatory networks with time-varying delay. The time delays $d(t)$ representing the translation delay and feedback regulation delay are time-varying. By using Itô's differential formula and Lyapunov stability theory, we have proposed a linear matrix inequality method to derive sufficient conditions under which the desired filters exist. We have also characterized the expression of the filter parameters and the decay rate $\beta>0$, and employed a simulation example to illustrate the effectiveness of the proposed results. It should be pointed out that it is not difficult to extend the main results in this paper to more complex and realistic systems, such as systems with nonlinearity constraints. Furthermore, although not directly applicable to time-delay systems, the traditional Kalman and Stratonovich filters may be useful for genetic regulatory networks with stochastic disturbances, and this is now under investigation.

\section{REFERENCES}

[1] A. Becskei and L. Serrano, Engineering stability in gene networks by autoregulation, Nature, vol. 405, pp. 590-593, 2000.

[2] S. Boyd, L. EI Ghaoui, E. Feron and V. Balakrishnan, Linear Matrix Inequalities in Systems and Control Theory, SIAM, Philadelphia, PA, 1994.

[3] B. S. Chen and Y. Wang, On the attenuation and amplification of molecular noise in genetic regulatory networks, BMC Bioinformatics, vol. 7, Art. no. 52, Feb. 2006.

[4] B. S. Chen and W. S. Wu, Robust filtering circuit design for stochastic gene networks under intrinsic and extrinsic molecular noises, Mathematical Bioscience, vol. 211, no. 2, pp. 342-355, 2008.

[5] B. S. Chen, Y. T. Chang and Y. C. Wang, Robust $H_{\infty}$ stabilization design in gene networks under stochastic molecular noises: fuzzy-interpolation approach, IEEE Transactions on System, Man and Cybernetics Part B: Cybernetics , vol. 38, no. 1 , pp. 25-42, 2008.

[6] T. Chen, H. He and G. Church, Modeling gene expression with differential equations, Proc. Pacific Symposium on Biocomputing, vol. 4, pp. 29-40, 1999. 


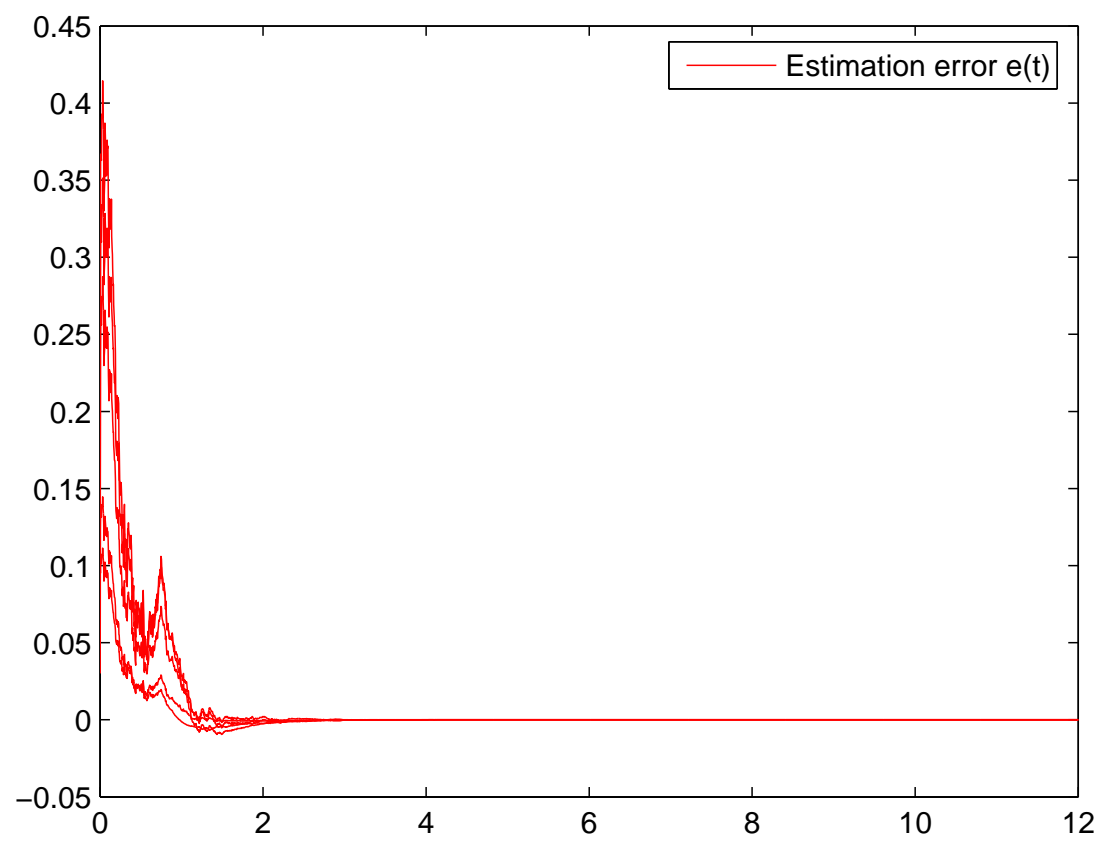

Fig. 2. Estimation error of $e(t)$

[7] L. Chen and K. Aihara, Stability of genetic regulatory networks with time delay, IEEE Trans. Circuits and Systems - Part I, vol. 49, pp. 602-608, 2002.

[8] D. Cook, A. Gerber and S. Tapscott, Modeling stochastic gene expression: implications for haploinsufficiency, Proc. the National Academy of Science, USA, vol. 95, pp. 15641-15646, 1998.

[9] P. D'haeseleer, X. Wen, S. Fuhrman and R. Somogyi, Linear modeling of mRNA expression levels during CNS development and injury, Proc. Pacific Symposium on Biocomputing, vol. 4, pp. 41-52, 1999.

[10] H. Gao, J. Lam and C. Wang, Robust energy-to-peak filter design for stochastic time-delay systems, Systems $\mathcal{E}$ Control Letters, vol. 55, no. 2, pp. 101-111, 2006.

[11] H. Gao, J. Lam and C. Wang, Induced $l_{2}$ and generalized $H_{2}$ filtering for systems with repeated scalar nonlinearities, IEEE Trans. Signal Processing, vol. 53, no. 11, pp. 4215-4226, 2005.

[12] H. Gao, C. Wang, Delay-dependent robust $H_{\infty}$ and $L_{2}-L_{\infty}$ filtering for a class of uncertain nonlinear time-delay systems, IEEE Trans. Automatic Control, vol. 48, no. 9, pp. 1631-1640, 2003.

[13] T. Gardner, C. Cantor and J. Collins, Construction of a genetic toggle switch in Escherichia coli, Nature, vol. 403, pp. 339-342, 2000.

[14] Y. He, Q. Wang, L. Xie and C. Lin, Further improvment of free-weighting matrices technique for systems with time-varying delay, IEEE Trans. Automatic Control, vol. 52, no. 2, pp. 293-299, 2007.

[15] S. Huang, Gene expression profilling, genetic networks, and cellular states: an integrating concept for tumorigenesis and drug discovery, Journal of Molecular Medicine, vol. 77, pp. 469-480, 1999.

[16] M. de Hoon, S. Imoto, K. Kobayashi, N. Ogasawara, and S. Miyano, Infering gene regulatory networks from time-ordered gene expression data of bacillus subtilis using differential equations, Proc. Pacific Symposium on Biocomputing, vol. 8, pp. 17-28, 2003.

[17] J. K. Hale, The theory of functional differential equations. New York: Springer-Verlag, 1977.

[18] J. Hasty, J. Pradines, M. Dolink and J. Collins, Biophysics Noise-based switches and amplifiers for gene expression, Proc. Natl Acad. Sci. vol. 97, no. 5, pp. 2075-2080, 2000.

[19] C. Li, L. Chen and K. Aihara, Synchronization of coupled nonidentical genetic oscillators, Phys. Biol., vol. 3, pp. 37-44, 2006.

[20] C. Li, L. Chen, and K. Aihara. Stability of genetic networks with sum regulatory logic: Lur'e system and LMI approach. IEEE Trans. Circuits and Systems - Part I, vol. 53, no. 11, pp. 2451-2458, 2006.

[21] H. Kushur, Stochastic Stability and Control, Academic Press, New York, 1976. 


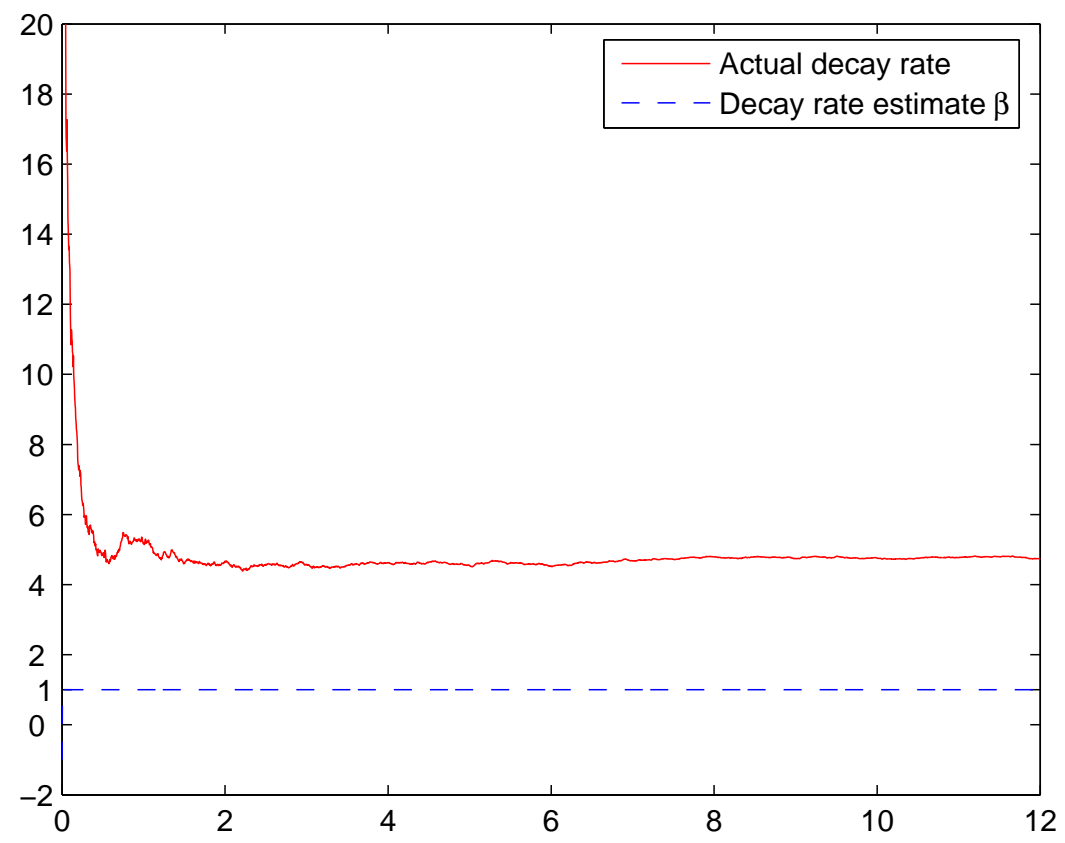

Fig. 3. Actual decay rate of $\bar{x}(t)$ and estimated decay rate $\beta$

[22] S. A. Kauffman, Metabolic stability and epigenesis in randomly constructed genetic nets, J. Theor. Biol., vol. 22, no. 3, pp. 437-467, 1969.

[23] S. A. Kauffman, The origins of Order, Oxford University Press, New York, 1993.

[24] X. Mao, Stochastic Differential Equations and Their Applications, Horwood Publishing Series in Mathematics and Applications, Horwood, Chichester, 1997.

[25] N. A. M. Monk, Oscillatory expression of Hes1, p53, and NF- $\kappa$ B driven by transcriptional time delays, Current Biol., vol. 13, pp. 1409-1413, 2003.

[26] J. Paulsson, Summing up the noise in gene networks, Nature, vol. 427, pp. 415-418, 2004.

[27] A. Pease, D. Solas, E. Sullivan, M. Cronin, C. Holmes, S. Fodor, Light-generated oligonucleotide arrays for rapid DNA sequense analysis, Proc. Natl. Acad. Sci. USA, vol. 91, pp. 5022-5026, 1994.

[28] F. Ren and J. Cao, Asymptotic and robust stability of genetic regulatory networks with time-varying delays, Neurocomputing, in press (doi:10.1016/j.neucom.2007.03.011)

[29] Z. Shu and J. Lam, Delay-dependent exponential estimates of stochastic neural networks with time delay, Proc. International Conference on Neural Information Processing, Part I, Lecture Notes in Computer Science, vol. 4232, pp. 332-341, 2006.

[30] P. Smolen, D. Baxter and J. Byrne, Mathematical modeling of gene networks, Neuron, vol. 26, pp. 567-580, 2000.

[31] P. Smolen, D. Baxter and J. Byrne, Modelling circadian oscillations with interlocking positive and negative feedback loops, J. Neurosci., vol. 21, pp. 6644-6656, 2001 .

[32] R. Thomas, Boolean formalization of genetic control circuits, J. Theor. Biol., vol. 42, no. 3, pp. 563-585, 1973.

[33] T. Tian and K. Burrage, stochastic neural network models for gene regulatory networks, Proc. IEEE Congress on Evolutionary Computation, pp. 162-169, 2003.

[34] Z. Wang, F. Yang, D. W. C. Ho and X. Liu, Robust $H_{\infty}$ filtering for stochastic time-delay systems with missing measurements, IEEE Trans. Signal Processing, vol. 54, No. 7, Jul. 2006, pp. 2579-2587.

[35] Z. Wang and K. Burnham, Robust filtering for a class of stochastic uncertain nonlinear time-delay systems via exponential state estimation, IEEE Trans. Signal Processing, vol. 49, no. 4, pp. 794-804, 2001.

[36] M. Wu, Y. He, J. She and G. Liu, Delay-dependent criteria for robust stability of time-varying delay systems, Automatica, vol. 40, no. 8, pp. 1435-1439, 2004.

[37] G. Wei, Z. Wang, H. Shu, K. Fraser and X. Liu, Robust filtering for gene expression time series data with variance constraints, Int. J. Computer Mathmatics, vol. 84, no. 5, pp. 619-633, 2007. 